\title{
The significance of presenteeism for the value of lost production: the case of rheumatoid arthritis
}

This article was published in the following Dove Press journal:

ClinicoEconomics and Outcomes Research

14 July 2010

Number of times this article has been viewed

\author{
Rikke Søgaard' \\ Jan Sørensen' \\ Louise Linde ${ }^{2}$ \\ Merete L Hetland ${ }^{2,3}$ \\ 'CAST - Centre for Applied Health \\ Services Research and Technology \\ Assessment, University of Southern \\ Denmark, Denmark; ${ }^{2}$ Department of \\ Rheumatology, Hvidovre and Glostrup \\ Hospitals, Denmark; ${ }^{3}$ The DANBIO \\ registry, Hvidovre and Glostrup \\ Hospitals, Denmark
}

\begin{abstract}
Lost production can be due to individuals' time lost to work (absenteeism), as well as their time at work with reduced productivity because of ill health (presenteeism). A sound methodological framework for the assessment of presenteeism remains to be established but given its significance, ignoring it would lead to severe underestimations, eg, in cost-of-illness studies. The objective of this study was to assess the empirical significance of absenteeism and presenteeism in terms of production loss using the case of rheumatoid arthritis (RA). Selected modules from the Health and Labor Questionnaire were applied in a cross-sectional study of 3,704 patients with RA. The costs of absenteeism and presenteeism were estimated using the Human Capital approach, and the impact of including multipliers adjusting for the productivity effect of a workers' absence or impaired presenteeism on societal productivity was demonstrated. RA-related absenteeism over the last 14 days was 22.31 hours (standard deviation [SD], 26.51) with a resulting cost of $€ 473$ (SD, 575) and $€ 762$ (SD, 926) depending on whether a multiplier was included. Presenteeism was found to affect 7.98 (SD, 3.24) working days over the last 14 days with a resulting cost of $€ 168$ (SD, 203) and $€ 203$ (SD, 245), again depending on whether a multiplier was included. Overall, this article demonstrates that the value of lost production due to RA could be subject to an almost factor 2 increase if productivity effects of presenteeism and general multipliers are included.
\end{abstract}

Keywords: work limitations, Health and Labor Questionnaire, productivity, production loss

\section{Introduction}

Rheumatoid arthritis (RA) is the most common inflammatory arthritis in adults affecting up to $1 \%$ of the population worldwide. It is a highly disabling disease that has been found to significantly depress health-related quality of life not only compared with the normal population but also with other musculoskeletal diseases, eg, symptomatic osteoarthritis, low back pain, and soft tissue disorders. ${ }^{1,2}$ The economic burden of RA to society has been estimated at $€ 45.3$ billion in Europe and $€ 41.6$ billion in the United States due to both direct health care costs and indirect costs of functional disability. ${ }^{3}$ In fact, about every second patient with RA can expect work disability within 10 years after disease onset, and thus, the costs of societal production loss are assumed to account for more than $50 \%$ of the total costs of the disease. ${ }^{4}$ Some of the more recent estimates of the value of lost production range from $€ 5,076$ to $€ 10,900$ per patient per year when using the Human Capital approach for evaluating production losses..$^{5-11}$

The value of lost production can be defined as the value of lost productivity and/or the costs incurred to maintain societal productivity as a result of a worker's ill health. ${ }^{12}$ As such at least two scenarios lead to productivity losses: short-term or long-term 
sickness leave (absenteeism) and reduced efficiency while working (impaired presenteeism, generally termed just presenteeism). In a systematic literature review from 2006, it was concluded that none of the identified studies assessing the value of lost production in RA had included the costs of presenteeism. ${ }^{13}$ Since then, however, a Canadian study across 383 employed individuals has been reported; productivity loss was measured in dimensions of RA-related absences, reduced performance, decreased working hours, job change, and work disability, and included in a model of the overall cost of production loss. ${ }^{14}$ Interestingly, presenteeism was found to account for $41 \%$ of total costs, whereas decreased work hours and absenteeism accounted for only $12 \%$ and $10 \%$, respectively. These findings indicate that the current estimates of the RA-related costs of production loss are severely underestimated.

The reason for presenteeism generally not being included could be methodological challenges of measuring on-the-job efficiency. ${ }^{15,16}$ Not only is the frequency and the duration of reduced productivity required to quantify events of presenteeism, disease-related deviations from the normal level of efficiency (optimally in terms of both quality and quantity of work) must be assessed too. ${ }^{17}$ This often means that asking workers is not sufficient for valid observations as most workers' productivity is interrelated with colleague workers' productivity due to, eg, team production and time sensitivity of individual outputs. Such effects have been examined in relation to absenteeism in which the health-related impact on productivity was found to be up to multiple times the wage of the absent worker, depending on the type of job characteristics. ${ }^{18}$ This means that simply measuring number of days lost to work and multiplying it by the daily wage rate would underestimate the value of the true productivity effect.

A recent contribution to the question of how to measure the effects of presenteeism on productivity was reported by Pauly et al ${ }^{19}$ who surveyed more than 800 employers across 12 industries and various job types. The idea of their work was not only to quantify the extent of impaired presenteeism for individual workers but also to estimate the effect of such to corporate productivity. For that purpose, the authors commented that managers are essentially and intrinsically the most appropriate respondents. The first parameter was measured in terms of percentage of fewer hours a worker provides in case of acute and chronic illness and found to an average $29 \%$ and $27 \%$, respectively. The second parameter, a multiplier expressing the impact to corporate productivity of a unit of presenteeism, was estimated between 1.12 and 1.71 for acute conditions and between 1.21 and 2.00 for chronic conditions, depending on the econometric model applied. The overall result of these estimations was expressed as the effect of presenteeism on the output of a whole department as a percentage of the wage of the worker suffering ill health.

The objective of this study was to assess the empirical significance of both absenteeism and presenteeism as sources of lost production using the case of rheumatoid arthritis. In addition to being one of the first studies in RA to have included presenteeism as a source of production loss, the novelty of the present contribution is the adaptation of multipliers of productivity effects from the recent literature, which are used to weigh original, patient-reported observations. The primary analysis of this study is restricted to patients who are active at the labor market as nonemployed patients obviously cannot contribute to on-the-job production loss. A secondary analysis then extends the study sample also to include patients who are inactive at the labor market to demonstrate the potential impact of including presenteeism on estimates of the societal value of production loss. The secondary analysis compares four approaches that are based on the Human Capital approach for valuation: with and without including presenteeism and with and without adapting multipliers to account for the fact that productivity effects of a worker's absence or presenteeism generally leads to a production loss, which is larger than the gross wage of that worker.

\section{Method}

\section{Patients and study design}

This study was part of a nationally representative crosssectional study examining the effects of biological and other treatments in RA..$^{20,21}$ The study was undertaken from June 2006 to July 2007 at 11 hospital-based rheumatologic clinics. All patients with an RA diagnosis as defined by the American College of Rheumatology 1987 criteria $^{22}$ were eligible for inclusion. Clinical data were recorded by physicians and registered in the nationwide DANBIO registry that monitors medical treatment of RA patients. ${ }^{23}$

Patients' self-reported ability to participate at the labor market was assessed using selected modules of the Health and Labor Questionnaire (HLQ). ${ }^{24}$ Module 1 was applied to assess patients' absence from paid work, and the descriptive part of Module 2 was applied to assess reduced productivity at paid work. Both modules ask the respondent about the 
compensation equivalent of the reduced productivity within the last 14 days.

\section{Primary parameters}

The primary parameters were 14-day prevalence of absenteeism (in hours) and presenteeism (yes/no). The number of hours of absenteeism was calculated from the patient-reported number of days (one day assumed to be equivalent to 7.4 hours) and half days (assumed to be equivalent to 3.7 hours) of reduced working time, sick leave due to RA, or sick leave due to other health problems. The prevalence of impaired presenteeism was reported on a Likert scale of "never," "sometimes," "often," and "always" across six dimensions of work: concentration, pace of work, need to be alone, ability to make decisions, delays in work progress, and ability to take over colleagues' work. Responses in presenteeism were dichotomized by collapsing "sometimes," "often," and "always" as an expression of presenteeism, whereas "never" expressed no presenteeism.

\section{Valuation of parameters}

The cost of absenteeism was estimated by the conventional approach of using workers' time cost to approximate the value of their productive input. National average age-matched and gender-matched gross salaries were converted to a cost per hour (annual estimate/12 months/160.33 hours per month), which was multiplied by the hours observed. The cost was estimated with and without the inclusion of a multiplier of 1.61 , which was adapted from Nicholson et $\mathrm{al}^{18}$ who defines the multiplier as the cost to (societal) production of an absence as a proportion of the absent worker's daily wage.

The cost of presenteeism was based on two assumptions due to the format of observations (proportion of respondents affected rather than exact amount of hours). First, presenteeism was assumed to impact productivity if a respondent reported problems in at least three of the six dimensions. Second, the magnitude of productivity effects was adapted from the study of Pauly et a ${ }^{19}$ who concludes an average impact of presenteeism on productivity of 0.27 for chronic health problems. Based on these assumptions, the value of production loss caused by presenteeism was calculated as events of presenteeism per half day of work, multiplied by national average age-matched and gender-matched gross salaries. Again, the cost was estimated with and without a multiplier; the estimate of 1.21 (applicable for chronic conditions) was adapted from Pauly et al. ${ }^{19}$
For the estimation of an annual value of production loss due to RA, four variants of the Human Capital approach was applied: basing estimates strictly on absenteeism (as is generally the case in the clinical literature), basing estimates on both absenteeism and presenteeism, and combining each of these with the inclusion of multipliers. Gross salaries approximated the value of time of patients in paid employment and patients on sick leave. Housewives' and students' time were valued using net salaries (their opportunity cost for deciding not to be in paid employment). It should be noted that this represents a secondary analysis for the purpose of examining the impact of a refined approach only (multiplier approach including also presenteeism) as it is based on the additional assumption that observations of 14-day prevalence can be extrapolated to a whole year.

All monetary values are in 2007-EUR.

\section{Sensitivity analysis}

This study was explorative in assessing the impact of different approaches for valuing productivity loss, rather than providing empirical estimates for adaptation in, eg, models of the costs of RA, and for that reason comprehensive sensitivity analysis was inappropriate. Sensitivity analysis was conducted for the parameter of presenteeism costs. In particular, the asumptions made to convert observed events of presenteeism per half day into a total number of hours and then into a total cost were tested. The wage rate used for valuation was kept constant in the sensitivity analysis. Two scenarios were tested. First, as the base-case scenario was fairly conservative in accepting on-the-job impairments only if observed in at least three of six dimensions of the presenteeism module, the impact of moving the cutpoint to impairments having effect if reported in only one dimension was tested. Second, as the base case did not allow the impact of presenteeism to vary in severity, the impact of grading it according to the number of dimensions reported was tested. This scenario included two subanalyses: one where the impact was allowed to vary from zero to a maximum, which is equal to the one adapted from Pauly et $\mathrm{al}^{19}$ (productivity deduction of 0.05 for every dimension affected) and another where the impact was allowed to vary from $0 \%$ to $200 \%$ (a productivity deduction of 0.10 for every dimension affected).

\section{Statistical analysis}

All parameters were reported as arithmetic means with standard deviations (SD) or as simple frequencies and percentages. The relation between absenteeism and presenteeism was examined using logistic regression and reported as odds ratios with 
confidence intervals (CI). A significance level of 0.05 was used, and all analysis was conducted in STATA version 11 (StataCorp, College Station, TX).

\section{Ethical considerations}

All patients were informed that participation was voluntary and independent of the provision of treatment modalities. Participation was accepted by filling in questionnaires. The study was approved by the Data Protection Agency whereas, according to Danish law, no ethical approval was needed for this study.

\section{Results}

Of the 3,704 patients who received the HLQ questionnaire, $1,900(51 \%)$ responded. The response was significantly different between employed and nonemployed patients as 1,049 ( $72 \%$ ) in paid employment responded to the questionnaire, whereas only 851 (38\%) not in paid employment responded. Accordingly, respondents were significantly younger and more likely to have college education than nonrespondents. Among those in paid work, which was the sample for the primary analysis, item responses (the extent to which individual items of the questionnaire were filled in by respondents; in the present context defined by at least one response in respective modules of the HLQ) were 945 (86\%) in the module relating to absenteeism and $969(88 \%)$ in the module relating to presenteeism.

Table 1 lists characteristics of the surveyed sample and the subgroup of patients in paid employment. For the latter group, the mean age was 49.94 (SD, 11.33) years and 74\% were females. The impact of disease on respondents' daily lives was reflected in a Health Assessment Questionnaire (HAQ) score of 0.38 (SD, 0.48) and in a health-related quality of life (EQ-5D) score of 0.75 (SD, 0.15). The current occupational status for patients in paid employment could be classified as $87 \%$ currently working, $7 \%$ on sick leave, and $6 \%$ were not classifiable.

\section{Absenteeism and presenteeism}

The average RA-related absenteeism over the last 14 days was 22.31 (SD, 26.51) hours of which sick leave accounted for 3.67 (16.06) hours and the remainder represented reduced working hours (see Table 2).

Presenteeism was reported over six dimensions: $43.23 \%$ reported problems with concentration at work, $56.77 \%$ reported problems with the pace of work, $36.24 \%$ reported need to be alone during working time, $24.43 \%$ reported problems with making decisions, $47.59 \%$ reported that their RA caused delays in work progress, and $36.26 \%$ reported to be less able to take over colleagues work (see Table 3 ).

It was noted that RA patients with an event of absenteeism are at higher risk of also experiencing impairments on the job. Among the 126 patients who responded to the presenteeism module of the questionnaire and who had at least one event of absenteeism, 113 (90\%) also reported presenteeism (defined as problems in any of the six dimensions). This was assessed using logistic regression, which estimated the odds ratio for presenteeism given all-cause absenteeism at $4.13(95 \%$ CI: 2.28-7.48) and the corresponding ratio for presenteeism given RA-related absenteeism at 15.29 (95\% CI: 3.72-62.86).

\section{The value of reduced productivity}

The total cost of RA-related absenteeism was estimated at $€ 473$ (SD, 575) and $€ 762$ (SD, 926) for a 14-day period,

Table I Baseline characteristics of the whole-study population of patients with rheumatoid arthritis $(\mathrm{N}=3,704)$ and the subgroup of patients in paid employment $(\mathrm{N}=1,455)$

\begin{tabular}{|c|c|c|c|c|}
\hline & $\begin{array}{l}\text { Whole-study } \\
\text { population }\end{array}$ & Response, $n$ & $\begin{array}{l}\text { Subgroup in paid } \\
\text { employment }\end{array}$ & Response, $\mathbf{n}$ \\
\hline Age, mean (SD) & $60.63(13.92)$ & 3,703 & $49.94(11.33)$ & $\mathrm{I}, 455$ \\
\hline Female, n (\%) & $2,780(75)$ & 3,704 & $\mathrm{I}, 08 \mathrm{I}(74)$ & $\mathrm{I}, 455$ \\
\hline HAQ score & & 2,407 & & 961 \\
\hline mean (SD) & $0.73(0.7 \mathrm{I})$ & & $0.48(0.53)$ & \\
\hline median (min; max) & $0.50(0.00 ; 3.00)$ & & $0.25(0.00 ; 2.85)$ & \\
\hline EQ-5D score, mean (SD) & $0.72(0.17)$ & 2,679 & $0.75(0.15)$ & $\mathrm{I}, 044$ \\
\hline Current ability to work, $\mathrm{n}(\%)$ & & 3,061 & & 1,049 \\
\hline Working full or part-time & $957(31)$ & & $916(87)$ & \\
\hline On sick leave & $126(4)$ & & $75(7)$ & \\
\hline On early retirement & $717(24)$ & & $0(0)$ & \\
\hline On pension & $947(31)$ & & $0(0)$ & \\
\hline Other & $314(10)$ & & $58(6)$ & \\
\hline
\end{tabular}

Abbreviations: SD, standard deviation; HAQ, Health Assessment Questionnaire; EQ-5D, EuroQol 5-dimensions. 
Table 2 Absenteeism-related production loss in 942 working patients with RA: observed I4-day prevalence and the estimated value of production loss based on the Human Capital approach

\begin{tabular}{llll}
\hline & Absenteeism in hours (SD) & \multicolumn{2}{l}{ Production loss in 2007-EUR (SD) } \\
\cline { 3 - 4 } & & Conventional approach & Multiplier approach \\
\hline Reduced working time & $18.64(22.90)$ & $394(494)$ & $634(795)$ \\
Sick-leave due to RA & $3.67(16.06)$ & $79(35 \mathrm{I})$ & $128(566)$ \\
Total RA-related absenteeism & $22.31(26.5 \mathrm{I})$ & $473(575)$ & $762(926)$ \\
Sick leave not related to RA & $2.16(11.04)$ & $46(24 I)$ & $75(388)$ \\
Total all-cause absenteeism & $24.47(27.83)$ & $520(606)$ & $837(976)$ \\
\hline
\end{tabular}

Note: The estimates of production loss represent (I) the conventional approach of using the workers' gross wage (age-matched and gender-matched national averages were used) as an approximation for the value of the output generated by a worker and (2) the conventional approach with the addition of adapting a multiplier of I.6I from Nicholson et al ${ }^{18}$ due to the cost of an absence often being larger than the absent worker's daily wage because absence generally affects colleague workers productivity and a replacement worker might not be readily available.

Abbreviations: RA, rheumatoid arthritis; SD, standard deviation.

depending on whether a multiplier was included. As the multiplier was a fixed parameter adapted from the literature of 1.61, the multiplier-based estimate is exactly $161 \%$ of the conventional. Table 2 further lists the total cost of all-cause absenteeism, which was found to be $€ 520$ (SD, 606) and $€ 837$ (SD, 976), again depending on whether a multiplier was included.

The costs of presenteeism were based on an average number of working days (within the 14-day period) of 7.98 (SD, 3.24) per patient and, in the base-case analysis, a definition of presenteeism affecting productivity only if reported in at least three of the six dimensions (see Table 3). Thus, 430 patients (44\%) reporting presenteeism in zero,

Table 3 Presenteeism-related production loss in 872 working patients with rheumatoid arthritis: observed 14-day prevalence and the estimated value of production loss based on the Human Capital approach

\begin{tabular}{ll}
\hline Dimension of presenteeism & $\begin{array}{l}\text { Patients reporting } \\
\text { presenteeism, \% }\end{array}$ \\
\hline Problems with concentration at work & 43.23 \\
Problems with the pace of work & 56.77 \\
Need to be alone during working time & 36.24 \\
Problems with making decisions & 24.43 \\
Experience delays in work progress & 47.59 \\
Less able to take over colleague's work & 36.26 \\
Summary estimates per patient & Mean (SD) \\
No. of dimensions reported & $2.42(2.18)$ \\
No. of working days reported & $7.98(3.24)$ \\
Production loss (2007-EUR) & \\
Conventional approach & $168(203)$ \\
Multiplier approach & $203(245)$ \\
\hline
\end{tabular}

Note: The estimates of production loss are based on an average reduction in worker productivity of 0.27 if presenteeism is reported in 3 or more dimensions; the conventional approach estimate uses the workers' gross wage (age-matched and gender-matched national averages were used) as an approximation for the value of the output generated by a worker, whereas the multiplier approach further includes a multiplier of $\mathrm{I} .2 \mathrm{I}$ from Pauly et al ${ }^{19}$ due to presenteeism often affecting departmental productivity rather than just output per worker. Abbreviation: SD, standard deviation. one, or two dimensions did not contribute to the cost of presenteeism in the base case, which was estimated at $€ 168$ (SD, 203) and $€ 203$ (SD, 245), again depending on whether a multiplier (of 1.21) was included. Sensitivity analysis moving the cutpoint from at least three dimensions to instead at least one dimension resulted in a cost increase to $€ 193$ (SD, 160) for the estimate not including the multiplier. Furthermore, the impact of instead allowing the severity of presenteeism to vary for the number of dimensions included was tested. A conservative scenario allowing the impact to vary from $0 \%$ to $30 \%$ reduced productivity as a percentage of the affected worker's wage lead to a reduced cost of $€ 117$ (SD, 129), whereas a more extreme scenario of allowing the impact to vary to a doubled maximum lead to an increased cost of $€ 233$ (SD, 257). As such, the impact of presenteeism is inherently subject to uncertainty, but it should be clear that at the same time it remains significant.

\section{The value of lost production per year due to rheumatoid arthritis}

As a supplement to the primary analysis and for comparison purposes, the sample of individuals in paid employment was extended to all patients $(n=3,704)$ for an overall estimate of the annual value of production loss due to RA. The impact of, first, including presenteeism as a source of production loss and, next, including multipliers to account for the effect of one worker's productivity on societal production is presented in Table 4. The purely absenteeism-based, conventional estimate amounted to $€ 14,920$ (SD, 18,585), and the more comprehensive estimate, including both absenteeism-related and presenteeism-related productivity effects, as well as multipliers, amounted to $€ 25,766$ (SD, 29,776), which is an almost factor 2 increase. 
Table 4 Comparison of variations of the Human Capital approach for valuing production loss: the case of working and nonworking patients with rheumatoid arthritis

\begin{tabular}{llll}
\hline & $\mathbf{n}$ & $\begin{array}{l}\text { Annual production } \\
\text { loss 2007-EUR (SD) }\end{array}$ & Range (min; max) \\
\hline $\begin{array}{l}\text { Conventional approach } \\
\quad \text { Absenteeism-based alone }\end{array}$ & & $14,920(18,585)$ & $(0 ; 58,155)$ \\
$\quad \begin{array}{l}\text { Absenteeism and presenteeism-based } \\
\text { Multiplier approach }\end{array}$ & 2,419 & $16,362(18,555)$ & $(0 ; 59,971)$ \\
$\quad$ Absenteeism-based alone & 2,419 & $24,021(29,922)$ & $(0 ; 93,629)$ \\
$\quad$ Absenteeism-based and presenteeism-based & 2,419 & $25,766(29,776)$ & $(0 ; 93,833)$ \\
\hline
\end{tabular}

Note: Although a greater " $n$ " could have been achieved for some rows (the ones requiring the least data), $n$ was defined as the largest sample for which all 4 estimates could be calculated. The estimates of production loss represent (I) the conventional approach of using the workers' gross wage (age-matched and gender-matched national averages were used) as an approximation for the value of the output generated by a worker and (2) the conventional approach with the addition of adapting a multiplier of $1.6 \mathrm{I}$ from Nicholson et $\mathrm{al}^{18}$ due to the cost of an absence often being larger than the absent worker's daily wage because absence generally affects colleague workers' productivity and a replacement for them might not be readily available.

Abbreviation: SD, standard deviation.

\section{Discussion}

This study assessed the 14-day prevalence of two main causes of reduced productivity: absenteeism (time lost from work) and presenteeism (on-the-job work limitations), in a large cohort of patients. The objective was to examine the role of presenteeism as a significant source of production loss as it is generally not included in economic evaluations or cost-of-illness studies in RA. The analysis furthermore included a second dimension of using multipliers to derive societal production effects from a single worker's absence or presenteeism, which is also not common practice in the applied literature. Overall, it was demonstrated that current estimates of the value of lost production due to RA could be significantly underestimated, in that productivity effects of presenteeism and general multipliers are not included.

Only one Canadian study has previously reported an estimate of the costs of presenteeism in arthritis. ${ }^{14}$ Based on a different approach than the one presented here, the authors concluded that the cost of presenteeism accounts for $41 \%$ of the cost of production loss. This is an even higher estimate than that of the present work, which was about 36\% (€168/€473). The difference between estimates could be due to differences in the measurement and valuation procedure (eg, different wage rates between Canada and Denmark) among other explanations. As a gold standard for assessing the cost of presenteeism has not been established, one empirical best bet can be as good as another.

The primary strength of this work in terms of the estimates of 14-day costs of absenteeism and presenteeism is the sample size of 3,704 patients. Examining effects that manifests in employed patients only requires more patients than is typically included in a clinical trial. Most RA patients will not be in paid employment, and even among those in paid employment, high response rates can be difficult to achieve due to respondents' reticence relating to ongoing claims for social benefits. Another strength is the adaptation of a fixed, but well-founded, weight of the effect of presenteeism on the productivity of a company rather than assuming that reduced productivity of one worker does not affect colleague workers. On the other hand, such adaptation of a fixed weight should be criticized for not being specific to the type of jobs held by patients with RA. Indeed, a first-best approach would be stochastic measurements, but as study units of patients are most often incapable of determining productivity of whole departments, it would require a study design including both patients and their managers as study units.

The response rate of only $51 \%$ could have lead to selection bias. In particular, as it appeared that nonresponders were significantly older and had more symptoms (higher HAQ score), the estimated average costs of absenteeism and presenteeism may have been underestimated, whereas the impact of selection bias to the significance of presenteeism relative to absenteeism is uncertain. Other potential bias include recall bias from the 14-day recall period used in the HLQ, as well as bias from the sampling procedure, ie, by sampling from a clinical database, only individuals with formally diagnosed RA are included.

The modules of the HLQ used for the present study have been validated as part of the original validation study for the instrument reported in $1996 .{ }^{24}$ The validation study was conducted in 995 individuals from the general population and in four disease-specific populations (migraine and three orthopedic diseases) and included issues of feasibility (response rates, missing values rates, completion time) and validity. The validity of responses of absenteeism was assessed by comparing self-reported values with data from 
a central office of statistics, whereas for assessing validity of responses concerning presenteeism, the authors commented that there was no gold standard. For that reason they compared the HLQ approach, which asks for a compensation equivalent of the reduced productivity, with the Osterhaus technique, ${ }^{25}$ which asks for a performance level and from that derives a productivity measure. An overall consensus was found.

In terms of the more explorative, methodological focus of the present study, the complexity of measuring on-the-job efficiency must be understood to determine validity of findings. The parameter of impaired presenteeism is a composite of at least three variables that must be assessed stochastically to estimate it: frequency, length, and efficiency. Frequency refers to the number of episodes of impaired presenteeism, which in the present study was assumed to be all working days (during the last 14 days) for respondents reporting work limitations. This is legitimized from to the wording of the questionnaire ("...think about the time when you were working...") although some respondents might have needed an option for reporting presenteeism in some days only, with the consequence of our estimates being in the upper end. Length refers to whether an episode of presenteeism affects the whole working day or some hours only. In this study, this was build in from the fixed parameter of 0.27 adapted from Pauly et $\mathrm{al}^{19}$ which we believe is a more valid path than attempting to handle it stochastically because such level of detail (whether impairments occur during 3 or 4 hours, say) would be complex to translate into effects on productivity. The third variable of efficiency refers to the effect of a worker's impairment on the firm's production (societal production). Although the two former variables are best assessed using workers as study units, efficiency requires managers or employers as study units. For that reason, this work adapted an impact weight from a study in more than 800 employers of various industries ${ }^{19}$ with the advantage of capturing both effects on colleague workers' productivity, as well as the monetary translation of it.

\section{Limitations}

This work attempted quantification of a construct for which no agreed-upon standard for assessment exists. The validated HLQ was applied for the measurement of a worker's on-the-job impairments although it has been demonstrated that alternative instruments could lead to different results. ${ }^{26}$ The translation of the measured impairments into productivity effects was based on a fixed weight from an external study, and the validity of such approach obviously relies on the external population (defined by chronic health problems) being representative for RA patients.

The use of a multiplier and the fact that a multiplier cannot be lower than one has been subject to criticism. Relating to absenteeism it has been suggested that workers often compensate for short-term absence during normal working hours. ${ }^{27}$ It is uncertain whether such compensation mechanisms lower productivity loss due to presenteeism too.

\section{Conclusion}

The implication of the present study should be a strengthened motivation for empirical focus and methodological developments in the measurement and valuation of presenteeism. For example, in a typical economic evaluation setup of assessing the value of a novel (and expensive) treatment regimen, that turns out effective in relation to functional ability, and thus in reducing the negative effects of presenteeism, the additional cost of such regimen could be outweighed by the saved costs of presenteeism. A second implication should be a critical stand in relation to the cost-of-illness literature in RA, which represents minimum estimates when not including the costs of presenteeism (and additional adjustment using a multiplier approach).

In conclusion, the value of lost production due to RA could be subject to an almost factor 2 increase if productivity effects of presenteeism and general multipliers are included. Results, however, remain uncertain until a sound methodological foundation for measuring presenteeism has been established.

\section{Acknowledgments}

Sørensen, Linde, and Hetland conceived the study. Søgaard conceived the economic analysis of the present paper, drafted the manuscript, and undertook all analysis. All authors participated in the revision of the manuscript and approved the final version.

Departments of rheumatology at the following hospitals contributed for data collection: Hvidovre Hospital, Gråsten Hospital, Hørsholm Hospital, Holbæk Hospital, Slagelse Hospital, Vejle Hospital, Hjørring Hospital, Bispebjerg Hospital, Frederiksberg Hospital, Herlev Hospital, Odense Hospital.

\section{Disclosure}

The authors declare that they have no competing interests. The study was financially supported by an unrestricted grant from the National Board of Health (j.nr. 0-204-3-9/2006) and an unrestricted grant from Schering-Plough. 


\section{References}

1. Salaffi F, De AR, Stancati A, Grassi W. Health-related quality of life in multiple musculoskeletal conditions: a cross-sectional population based epidemiological study. II. The MAPPING study. Clin Exp Rheumatol. 2005;23:829-839.

2. Salaffi F, Carotti M, Gasparini S, Intorcia M, Grassi W. The healthrelated quality of life in rheumatoid arthritis, ankylosing spondylitis, and psoriatic arthritis: a comparison with a selected sample of healthy people. Health Qual Life Outcomes. 2009;7:25.

3. Lundkvist J, Kastang F, Kobelt G. The burden of rheumatoid arthritis and access to treatment: health burden and costs. Eur J Health Econ. 2008;8 Suppl 2:S49-S60.

4. Jacobsson LT, Lindroth Y, Marsal L, Juran E, Bergstrom U, Kobelt G. Rheumatoid arthritis: what does it cost and what factors are driving those costs? Results of a survey in a community-derived population in Malmo, Sweden. Scand J Rheumatol. 2007;36:179-183.

5. Hallert E, Husberg M, Skogh T. Costs and course of disease and function in early rheumatoid arthritis: a 3-year follow-up (the Swedish TIRA project). Rheumatology (Oxford). 2006;45:325-331.

6. Huscher D, Merkesdal S, Thiele K, Zeidler H, Schneider M, Zink A. Cost of illness in rheumatoid arthritis, ankylosing spondylitis, psoriatic arthritis and systemic lupus erythematosus in Germany. Ann Rheum Dis. 2006;65:1175-1183.

7. Kobelt G, Sobocki P, Mulero J, Gratacos J, Pocovi A, Collantes-Estevez E. The burden of ankylosing spondylitis in Spain. Value Health. 2008; 11:408-415.

8. Kobelt G, Woronoff AS, Richard B, Peeters P, Sany J. Disease status, costs and quality of life of patients with rheumatoid arthritis in France: the ECO-PR study. Joint Bone Spine. 2008;75:408-415.

9. Merkesdal S, Ruof J, Schoffski O, Bernitt K, Zeidler H, Mau W. Indirect medical costs in early rheumatoid arthritis: composition of and changes in indirect costs within the first three years of disease. Arthritis Rheum. 2001;44:528-534.

10. Puolakka K, Kautiainen H, Pekurinen M, et al. Monetary value of lost productivity over a five year follow up in early rheumatoid arthritis estimated on the basis of official register data on patients' sickness absence and gross income: experience from the FIN-RACo trial. Ann Rheum Dis. 2006;65:899-904.

11. Wolfe F, Michaud K, Choi HK, Williams R. Household income and earnings losses among 6,396 persons with rheumatoid arthritis. J Rheumatol. 2005;32:1875-1883.

12. Brouwer WB, Koopmanschap MA. How to calculate indirect costs in economic evaluations. Pharmacoeconomics. 1998;13:563-569.

13. Burton W, Morrison A, Maclean R, Ruderman E. Systematic review of studies of productivity loss due to rheumatoid arthritis. Occup Med (Lond). 2006;56:18-27.

14. Li X, Gignac MA, Anis AH. The indirect costs of arthritis resulting from unemployment, reduced performance, and occupational changes while at work. Med Care. 2006;44:304-310.
15. Escorpizo R, Bombardier C, Boonen A, et al. Worker productivity outcome measures in arthritis. J Rheumatol. 2007;34:1372-1380.

16. Schultz AB, Chen CY, Edington DW. The cost and impact of health conditions on presenteeism to employers: a review of the literature. Pharmacoeconomics. 2009;27:365-378.

17. Koopmanschap M, Burdorf A, Jacob K, Meerding WJ, Brouwer W, Severens H. Measuring productivity changes in economic evaluation: setting the research agenda. Pharmacoeconomics. 2005;23:47-54.

18. Nicholson S, Pauly MV, Polsky D, Sharda C, Szrek H, Berger ML. Measuring the effects of work loss on productivity with team production. Health Econ. 2006;15:111-123.

19. Pauly MV, Nicholson S, Polsky D, Berger ML, Sharda C. Valuing reductions in on-the-job illness: 'presenteeism' from managerial and economic perspectives. Health Econ. 2008;17:469-485.

20. Linde L, Sorensen J, Ostergaard M, et al. What factors influence the health status of patients with rheumatoid arthritis measured by the SF-12v2 Health Survey and the Health Assessment Questionnaire? J Rheumatol. 2009;36:2183-2189.

21. Linde L, Sorensen J, Ostergaard M, Horslev-Petersen K, Hetland ML. Does clinical remission lead to normalization of EQ-5D in patients with RA and is the selection of remission criteria important? A comparative study of 3156 patients and 16136 individuals from the general population [submitted]. J Rheumatol. 2010;37:285-290.

22. Arnett FC, Edworthy SM, Bloch DA, et al. The American Rheumatism Association 1987 revised criteria for the classification of rheumatoid arthritis. Arthritis Rheum. 1988;31:315-324.

23. Hetland ML, Lindegaard HM, Hansen A, et al. Do changes in prescription practice in patients with rheumatoid arthritis treated with biological agents affect treatment response and adherence to therapy? Results from the nationwide Danish DANBIO Registry. Ann Rheum Dis. 2008;67:1023-1026.

24. van Roijen L, Essink-Bot ML, Koopmanschap MA, Bonsel G, Rutten FF. Labor and health status in economic evaluation of health care. The Health and Labor Questionnaire. Int J Technol Assess Health Care. 1996;12:405-415.

25. Osterhaus JT, Gutterman DL, Plachetka JR. Healthcare resource and lost labour costs of migraine headache in the US. Pharmacoeconomics. 1992;2:67-76.

26. Brouwer WB, Koopmanschap MA, Rutten FF. Productivity losses without absence: measurement validation and empirical evidence. Health Policy. 1999;48:13-27.

27. Jacob-Tacken KH, Koopmanschap MA, Meerding WJ, Severens JL. Correcting for compensating mechanisms related to productivity costs in economic evaluations of health care programmes. Health Econ. 2005; $14: 435-443$.
ClinicoEconomics and Outcomes Research

\section{Publish your work in this journal}

ClinicoEconomics \& Outcomes Research is an international, peerreviewed open-access journal focusing on Health Technology Assessment, Pharmacoeconomics and Outcomes Research in the areas of diagnosis, medical devices, and clinical, surgical and pharmacological intervention. The economic impact of health policy and health systems

\section{Dovepress}

organization also constitute important areas of coverage. The manuscript management system is completely online and includes a very quick and fair peer-review system, which is all easy to use. Visit http://www.dovepress.com/testimonials.php to read real quotes from published authors. 